SCIENTIFIC REPORT

\title{
Brimonidine purite $0.15 \%$ versus dorzolamide $2 \%$ each given twice daily to reduce intraocular pressure in subjects with open angle glaucoma or ocular hypertension
}

\author{
E D Sharpe, D G Day, C J Beischel, J S Rhodes, J A Stewart, W C Stewart
}

Br J Ophthalmol 2004;88:953-956. doi: 10.1136/bjo.2003.032979

Background/aims: To evaluate the efficacy of brimonidine purite versus dorzolamide given twice daily in primary open angle glaucoma or ocular hypertensive subjects.

Methods: In this double masked, multicentre, prospective, crossover comparison 33 subjects were randomised to brimonidine purite or dorzolamide for the first 4 week treatment period after a 4 week washout. Subjects began the opposite treatment for the second 4 week period after another 4 week washout. Intraocular pressure (IOP) was measured at 08:00 (trough) and 10:00, 18:00, and 20:00 hours after dosing at each baseline and at the end of each treatment period.

Results: The baseline diurnal IOP was 22.9 (SD 2.8) for brimonidine purite and 22.2 (SD 2.4) $\mathrm{mm} \mathrm{Hg}$ for dorzolamide. The trough IOP following 4 weeks of therapy was 21.0 (SD 3.7) for brimonidine purite and 21.0 (SD 3.1) mm Hg for dorzolamide $(p=0.90)$. The mean diurnal IOP was 19.3 (SD 3.1) for brimonidine purite and 19.8 (SD 2.4) $\mathrm{mm} \mathrm{Hg}$ for dorzolamide $(p=0.46)$. Dorzolamide caused more ocular stinging upon instillation $(n=8)$ than brimonidine purite $(n=1)(p=0.02)$. No statistical differences existed between groups for systemic adverse events.

Conclusions: This study suggests that brimonidine purite and dorzolamide each given twice daily have similar efficacy in primary open angle glaucoma or ocular hypertensive subjects. However, a trend was observed at 10:00 of greater brimonidine purite efficacy compared with dorzolamide.

$\mathrm{D}$ orzolamide (Trusopt, Merck \& Co, Inc, Blue Bell, PA, USA) and brimonidine (Alphagan, Allergan Inc, Irvine, CA, USA) are both popular antiglaucoma agents that are often used as adjunctive medications although they both may be prescribed as a monotherapy. When these agents have been evaluated apart from each other in clinical studies the monotherapy trough pressure reduction for both brimonidine and dorzolamide have been similar. ${ }^{1-8}$ In a direct comparison of both agents given three times daily, Stewart and associates showed similar morning trough (14\%) and peak (23-26\%) efficacy of brimonidine and dorzolamide. ${ }^{9}$

Recently a new formulation, brimonidine purite (Alphagan-P, Allergan Inc), was released which contains brimonidine as the active ingredient in the $0.15 \%$ concentration with purite as the preservative. Unfortunately few ocular hypotensive efficacy data exist regarding brimonidine purite compared with dorzolamide given twice daily.

In this present study we evaluated the use of brimonidine purite versus dorzolamide each given twice daily in subjects with primary open angle, exfoliation, or pigment dispersion glaucoma, or ocular hypertension in a prospective, randomised, double masked, crossover comparison.

\section{METHODS}

\section{Subjects}

We included in this study subjects with the following criteria: age 18 years or older; a clinical diagnosis of primary open angle, exfoliation, or pigment dispersion glaucoma, or ocular hypertension in at least one eye (study eye); intraocular pressure (IOP) controllable on one drug treatment in the study eye(s) in such a way that assured clinical stability of vision and the optic nerve throughout the trial; baseline IOP between 22-32 mm Hg inclusive in the study eye(s) (in eyes not included in the study IOP must have been controllable on no pharmacologic treatment or on the study medicine only); best corrected visual acuity of 20/200 or better in the study eye(s), and a mean diurnal pressure (average of the four measured time points) of $20-30 \mathrm{~mm} \mathrm{Hg}$ inclusive.

Subjects were excluded from this study for the following reasons: any abnormality preventing reliable applanation tonometry in the study eye(s); any opacity or subject uncooperativeness that restricted adequate examination of the ocular fundus or anterior chamber in the study eye; any concurrent infectious/non-infectious conjunctivitis, keratitis, or uveitis in either eye; any history of allergic hypersensitivity or poor tolerance to any components of the preparations used in this trial including sulfa medication; females of childbearing potential not using reliable means of birth control; pregnant or lactating females; any clinically significant, serious, or severe medical or psychiatric condition; participation in any investigational drug or device trial within the previous 30 days before the baseline (visit 2) of this trial; intraocular conventional surgery or laser surgery within two months of the trial in the study eye(s), risk of visual field or visual acuity worsening as a consequence of participation in the trial (according to the investigator's best judgment); inability to understand the trial procedures and thus inability to give informed consent; any anticipated change in systemic hypotensive therapy during the trial; progressive retinal or optic nerve disease apart from glaucoma, and current treatment with monoamine oxidase inhibitors.

\section{Procedures and assessments}

Following the signing of an institutional review board approved informed consent document, subjects underwent the following screening assessments: ocular and systemic history, Early Treatment Diabetic Retinopathy Study (ETDRS) visual acuity, slit lamp biomicroscopy, Goldmann applanation tonometry, gonioscopy, dilated fundus examination, and a Humphrey visual field (programme 24-2) (Humphrey Instruments, Dublin, CA, USA). Eligible subjects then were instructed to discontinue their current glaucoma 
medication and return to clinic after an appropriate washout period (that is, $\beta$-blockers, 4 weeks; prostaglandins, 6 weeks).

At visit 2 the subjects underwent a baseline examination between 07:00 and 09:00 which included-as did all subsequent examinations-ETDRS visual acuity, slit lamp biomicroscopy, and Goldmann applanation tonometry including: diurnal pressure measurements at 10:00, 18:00, and 20:00 hours after the morning trough (08:00) reading. At the end of the baseline examination, if the inclusion untreated IOP was met $(22-32 \mathrm{~mm} \mathrm{Hg}$ inclusive in at least one eye at 08:00 and $20-30 \mathrm{~mm} \mathrm{Hg}$ inclusive diurnal pressure), subjects were randomised either to brimonidine purite $0.15 \%$ or dorzolamide $2 \%$ to be instilled one drop in the study eye(s) at 08:00 and 20:00. Each bottle had masked labelling so the subject did not know which medicine he or she was taking. Only a dosing coordinator was allowed to observe the masked bottle during the study. Other study personnel and the investigators were masked to the study medicine.

Subjects returned after four weeks for the end of period 1 examination (visit 3, week 4). For both end of period evaluations the 10:00, 18:00, and 20:00 hour time points were measured after the trough (08:00) examination and dosing of the study medication. The period 1 medication then was discontinued and subjects underwent another 4 week washout. Subjects returned for period 2 (visit 4, week 8 ) and were initiated on the second medication after a trough (08:00) examination and diurnal IOP measurements. Subjects returned after four more weeks (visit 5, week 12) for the end of period 2 measurements and exit.

\section{Statistics}

All data analyses were two sided and an 0.05 alpha level used. The primary efficacy variable was the diurnal IOP difference at visit 3 and 5 . This was analysed by a paired $t$ test for intragroup analysis. ${ }^{10}$ An average eye analysis was used. The secondary efficacy variable, the IOPs at each individual time point, were also analysed by a paired $t$ test. ${ }^{10}$ This study provided an $80 \%$ power that a $1.5 \mathrm{~mm} \mathrm{Hg}$ difference could be excluded between groups if 27 subjects completed the study. A standard deviation of $2.8 \mathrm{~mm} \mathrm{Hg}$ was assumed. ${ }^{11-13}$ Changes in both the "study baseline" (visit 2 used as baseline) and the "period baseline" (the baseline of each individual treatment used at visits 2 and 4) were also analyded by a paired $t$ test. Visual acuity was analysed by a paired $t$ test. Adverse events were evaluated with a McNemar test. $^{14}$

\section{RESULTS}

\section{Subjects}

We enrolled 33 subjects in this crossover comparison. Seventeen had ocular hypertension, 14 had primary open angle glaucoma, one had pseudoexfoliation glaucoma, and one had pigment dispersion glaucoma. Fifteen subjects were

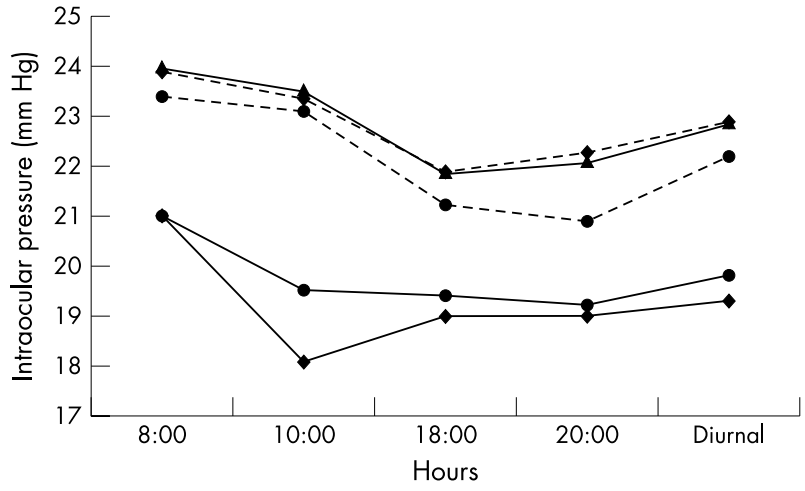

Figure 1 Diurnal mean intraocular pressures for common baseline at week 0 (triangles), brimonidine purite at baseline (dotted diamonds), and at week 4 (diamonds), and dorzolamide at baseline (dotted circles) and at week 4 (circles).

male and 18 were female; 14 were white and 19 were African-American. The mean age was 64.3 (SD 11.0) years.

\section{Intraocular pressure}

Period baseline diurnal IOP for brimonidine purite was 22.9 (SD 2.8) and 22.2 (SD 2.4) $\mathrm{mm} \mathrm{Hg}$ for dorzolamide (table 1, fig 1). The reduction in IOP from baseline was statistically significant for both treatments for each time point and for the diurnal curve. Also, there were no significant differences at any individual time point or for the diurnal curve between medicines with the reduction of pressure from visit 2 (that is, study baseline) (table 1 , fig 1 ).

This study found that the 08:00 trough IOP following 4 weeks of treatment was 21.0 (SD 3.7) for brimonidine purite and 21.0 (SD 3.1) $\mathrm{mm} \mathrm{Hg}$ for dorzolamide $(\mathrm{p}=0.90)$. The diurnal pressure was 19.3 (SD 3.1) for brimonidine purite and 19.8 (SD 2.4) $\mathrm{mm} \mathrm{Hg}$ for dorzolamide $(\mathrm{p}=0.46)$. There was no statistical difference between treatments for any individual time point or for the diurnal curve.

However, when compared to each medicine's own period baseline a greater reduction was observed for brimonidine purite than dorzolamide at 10:00 $(\mathrm{p}=0.03)$ and 20:00 $(p=0.04)$ hours after morning dosing and for the diurnal curve $(\mathrm{p}=0.03) \quad$ (table 2$)$. When period baselines were examined for a crossover effect, none was observed for brimonidine (that is, visits 2 and 4 baselines showed similar IOPs) (table 3). In contrast, the period baseline was lower for dorzolamide at visit 4 than visit 2 (table 3 ).

\section{Adverse events}

Unsolicited ocular adverse events are shown in table 4. There was a significantly increased incidence of stinging/burning with dorzolamide $(\mathrm{n}=8)$ compared with brimonidine purite $(\mathrm{n}=1) \quad(\mathrm{p}=0.02)$. No other statistical differences were observed. Unsolicited systemic side effects reported are

\begin{tabular}{|c|c|c|c|c|c|c|}
\hline & $\begin{array}{l}\text { Baseline } \\
\text { (Visit 2) }^{*}\end{array}$ & $\begin{array}{l}\text { Brimonidine purite } \\
\text { Period baseline } \neq\end{array}$ & $\begin{array}{l}\text { Active } \\
\text { TX }\end{array}$ & $\begin{array}{l}\text { Dorzolamide } \\
\text { Period baselineł }\end{array}$ & $\begin{array}{l}\text { Active } \\
\text { TX }\end{array}$ & p Value \\
\hline Trough & $24.0(2.2)$ & 23.9 (3.5) & $21.0(3.7)$ & $23.4(2.7)$ & $21.0(3.1)$ & 0.90 \\
\hline $10: 00$ & $23.5(2.9)$ & 23.4 (3.0) & 18.1 (4.1) & 23.1 (3.0) & $19.5(2.8)$ & 0.10 \\
\hline $18: 00$ & $21.9(2.8)$ & 21.9 (3.2) & 19.0 (3.1) & $21.2(3.1)$ & $19.4(3.0)$ & 0.59 \\
\hline $20: 00$ & $22.1(2.6)$ & $22.3(3.1)$ & $19.0(3.1)$ & 20.9 (3.0) & $19.2(3.1)$ & 0.69 \\
\hline Diurnal & $22.9(2.2)$ & $22.9(2.8)$ & $19.3(3.1)$ & $22.2(2.4)$ & $19.8(2.4)$ & 0.46 \\
\hline
\end{tabular}

*Study baseline $=$ average of all pressures at visit 2 before beginning any TX $\ddagger$ Period baseline $=$ average pressures for each TX at the beginning of each period (visits 2 and 4). $\mathrm{TX}=$ treatment 


\begin{tabular}{llll}
$\begin{array}{l}\text { Table } 2 \\
\text { (mm Hg) }\end{array}$ & Mean (SD) reduction from period baseline \\
\hline & Brimonidine purite & Dorzolamide & p Value \\
\hline Trough & $2.8(2.8)$ & $2.5(2.7)$ & 0.63 \\
$10: 00$ & $5.3(3.6)$ & $3.6(2.5)$ & 0.03 \\
$18: 00$ & $2.9(3.4)$ & $1.8(3.1)$ & 0.17 \\
$20: 00$ & $3.3(2.7)$ & $1.7(3.6)$ & 0.04 \\
Diurnal & $3.6(2.3)$ & $2.4(2.0)$ & 0.03 \\
\hline
\end{tabular}

shown in table 5. There were no serious adverse events or discontinued subjects during the trial. Treated visual acuities were statistically similar between groups $(p=0.68)$.

\section{DISCUSSION}

Dorzolamide $2 \%$ is a topical carbonic anhydrase inhibitor that was released to provide a safer alternative to the oral carbonic anhydrase inhibitor, acetazolamide, in the treatment of primary open angle glaucoma or ocular hypertension. Dorzolamide has been a popular adjunctive agent and is not infrequently used as monotherapy. Dosing for dorzolamide is labelled three times daily, but it may be tried twice daily. ${ }^{4}$ Dorzolamide reduces IOP at trough by $10-26 \%$ as a monotherapy agent ${ }^{4-8}$ and has eliminated most of the systemic symptoms commonly associated with acetazolamide. To date, bone marrow depression has been almost non-existent and kidney stone incidence has not been above the expected level for the general population. ${ }^{8}$ Several side effects do occur commonly with dorzolamide, however, including bitter taste, stinging, ocular pain on instillation, and conjunctival hyperaemia. ${ }^{78} 15$

Brimonidine $0.2 \%$ was commercially released and is a highly selective $\alpha_{2}$ adrenergic agonist. It reduces IOP at the 08:00 trough level approximately $15-16 \%$ from baseline. $^{2}{ }^{3} 16{ }^{17}$ Like dorzolamide, dosing is labelled as three times daily. It has become a popular adjunctive agent for glaucoma and, when prescribed, it is frequently given as monotherapy. However, brimonidine also may cause side effects, including ocular intolerance with an incidence of about $10 \%$ presenting three months or more after starting treatment. ${ }^{16}$ Also, systemic side effects of dry mouth, fatigue, and blood pressure changes may occur. ${ }^{18} 19$

Recently a new formulation of brimonidine, brimonidine purite $0.15 \%$, was released that contains purite as the preservative in a $0.15 \%$ concentration. Katz showed that when given three times a day these medications provide statistically similar morning trough and peak pressure results. ${ }^{2}$ Unfortunately, little peer reviewed information

Table 3 Mean (SD) period baseline pressures separated by treatment period ( $\mathrm{mm} \mathrm{Hg}$ )

\begin{tabular}{lllll}
\hline & & $\begin{array}{l}\text { Period baseline, } \\
\text { visit 2 }\end{array}$ & $\mathbf{N}$ & $\begin{array}{l}\text { Period baseline, } \\
\text { visit 4 }\end{array}$ \\
\hline $\begin{array}{l}\text { Brimonidine purite } \\
\text { Trough }\end{array}$ & 17 & $23.9(4.4)$ & 16 & $23.9(2.4)$ \\
10:00 & 17 & $23.4(3.3)$ & 16 & $23.3(2.8)$ \\
18:00 & 17 & $22.2(3.1)$ & 16 & $21.6(3.4)$ \\
20:00 & 17 & $22.6(2.9)$ & 16 & $21.9(3.1)$ \\
Diurnal & 17 & $23.0(3.1)$ & 16 & $22.7(2.5)$ \\
Dorzolamide & & & & \\
Trough & 16 & $24.2(2.3)$ & 17 & $22.8(3.1)$ \\
10:00 & 16 & $23.8(3.0)$ & 17 & $22.4(2.9)$ \\
18:00 & 16 & $22.2(2.1)$ & 17 & $20.5(3.8)$ \\
20:00 & 16 & $22.1(2.1)$ & 17 & $19.7(3.3)$ \\
Diurnal & 16 & $23.1(1.9)$ & 17 & $21.4(2.7)$ \\
\hline
\end{tabular}

Table 4 Unsolicited ocular adverse events (number of subjects with such an event; two or more events indicated)

\begin{tabular}{llll}
\hline & $\begin{array}{l}\text { Brimonidine } \\
\text { purite }\end{array}$ & Dorzolamide & p Value \\
\hline Conjunctival hyperaemia & 4 & 5 & $>0.999$ \\
Burning upon instillation & 1 & 8 & 0.020 \\
Blurriness after instillation & 2 & 3 & $>0.999$ \\
Ocular pain & 2 & 1 & 0.500 \\
Superficial punctate & 1 & 1 & $>0.999$ \\
keratitis & 1 & 1 & $>0.999$ \\
Ocular swelling & 1 & & \\
\hline
\end{tabular}

exists regarding the direct comparative efficacy and safety of brimonidine purite and dorzolamide within the same subject population.

The purpose of this trial was to compare the efficacy and safety of dorzolamide and brimonidine each given twice daily in subjects with primary open angle glaucoma or ocular hypertension.

This study showed that the IOP at each individual time point evaluated and for the diurnal curve (average of the four time points) were statistically equal between the brimonidine purite and dorzolamide treatment groups. In addition, each treatment group showed a statistical reduction from baseline at each time point and for the diurnal curve from the medicine's own period baseline (visits 2 and 4) or from the study baseline (visit 2 ).

However, when treated pressures were compared with the period baseline there was a greater statistical reduction with brimonidine than dorzolamide at 10:00 and 20:00 hours after dosing and for the diurnal curve. The reason for little difference in the absolute pressure levels between treatments, but a reduced diurnal period baseline for dorzolamide (about $0.7 \mathrm{~mm} \mathrm{Hg}$ ) compared with brimonidine, was not completely clear.

One explanation could be derived from examining how the period baselines were divided out between visits 2 and 4 . The period baselines between these visits for brimonidine were similar. Also, baseline pressures between subjects treated with dorzolamide or brimonidine purite at visit 2 were similar. However, the baseline diurnal pressures for subjects treated with dorzolamide at visit 4 were on average $1.7 \mathrm{~mm} \mathrm{Hg}$ less than subjects treated with dorzolamide at visit 2. Consequently, there may have been a period crossover effect despite the 4 week washout of brimonidine that influenced subjects treated with dorzolamide in period 2 .

Washout times for topical $\alpha$ adrenergic agonists in clinical protocols are generally assumed to be $2-3$ weeks. ${ }^{20}$ However, Stewart and associates recently evaluated the washout effect in 15 subjects treated with brimonidine therapy. ${ }^{21}$ Pressures were measured twice weekly until subjects returned to baseline. Four weeks after stopping brimonidine therapy five

Table 5 Unsolicited systemic adverse events (number of subjects with such an event; two or more events indicated)

\begin{tabular}{lllc}
\hline & $\begin{array}{l}\text { Brimonidine } \\
\text { purite }\end{array}$ & Dorzolamide & p Value \\
\hline Bitter taste upon instillation & 1 & 3 & 0.25 \\
Benign prostatic hyperplasia & 1 & 1 & $>0.999$ \\
Left leg injury & 1 & 1 & $>0.999$ \\
$\begin{array}{l}\text { Non-insulin dependent } \\
\text { diabetes }\end{array}$ & 1 & 1 & $>0.999$ \\
Worsening of osteoarthritis & 1 & 1 & $>0.999$ \\
\hline
\end{tabular}


subjects still had a reduction from baseline in their worse eye that caused an average reduction in pressure of $1.7 \mathrm{~mm} \mathrm{Hg}$ for all 15 subjects. ${ }^{21}$ Consequently, it is possible that a longer washout time for $\alpha$ agonists is required in subjects undergoing a second baseline. According to the above study, a brimonidine crossover effect should not have occurred eight weeks following therapy based on the previous data at the end of period 2 (visit 5).

Nonetheless, at 10:00 brimonidine purite showed a trend of greater efficacy compared with dorzolamide. These data are consistent with the known strong peak effect of brimonidine two to four hours after dosing. ${ }^{32}$

Ocular events were similar between groups except for more ocular stinging with dorzolamide. This finding is consistent with past studies. ${ }^{23-25}$ Systemic side effects were similar between groups. No subjects in this study discontinued a treatment period early and, overall, both medicines were well tolerated in this study.

This study did not evaluate the efficacy and safety of dorzolamide and brimonidine purite given as adjunctive therapy or as mono or adjunctive therapy prescribed per label three times daily. In addition, this study was not long enough to capture potential allergic effects associated with brimonidine purite. Further evaluation may clarify the specific advantages or disadvantages of these medicines in the stepwise therapy of glaucoma.

This study suggests that there is similar efficacy and safety between monotherapy treatment with brimonidine purite and dorzolamide each given twice daily in primary open angle glaucoma or ocular hypertensive subjects. However, a trend was observed at 10:00 of greater brimonidine purite efficacy compared with dorzolamide.

\section{Authors' affiliations}

E D Sharpe, Ophthalmology Consultants, Mt Pleasant, SC, USA

D G Day, Omni Eye Services, Atlanta, GA, USA

C J Beischel, Kulze \& Beischel, Charleston, SC, USA

J S Rhodes, J A Stewart, W C Stewart, Pharmaceutical Research Network, LLC, Charleston, SC, USA

W C Stewart, Carolina Eye Institute at the University of South Carolina School of Medicine, Columbia, SC

Supported with an unrestricted grant from Merck, Inc, USA.

Correspondence to: William C StewartMD, Pharmaceutical Research Network, LLC, 1639 Tatum Street, Charleston, SC 29412-2464, USA; prnc@bellsouth.net

Accepted for publication 1 October 2003

\section{REFERENCES}

1 Orzalesi N, Rossetti L, Invernizsi T, et al. Effect of timolol, latanoprost, and dorzolamide on circadian IOP in glaucoma or ocular hypertension. Invest Ophthalmol Vis Sci 2000;41:2566-73.
2 Katz LJ. Twelve-month evaluation of brimonidine-purite versus brimonidine in patients with glaucoma or ocular hypertension. J Glaucoma 2002;11:119-26.

3 Schuman JS. Clinical experience with brimonidine $0.2 \%$ and timolol $0.5 \%$ in glaucoma and ocular hypertension. Surv Ophthalmol 1996;41:S27-S37.

4 Lippa EA, Carlson LE, Ehinger B, et al. Dose response and duration of action of dorzolamide, a topical carbonic anhydrase inhibitor. Arch Ophthalmol 1992;110:495-9.

5 Lippa EA, Schuman JS, Higginbotham EJ, et al. MK-507 versus sezolamide: Comparative efficacy of two topically active carbonic anhydrase inhibitors. Ophthalmology 1991;98:308-13.

6 Wilkerson J, Cyrlin M, Lippa EA, et al. Four-week safety and efficacy study of dorzolamide, a novel, active topical carbonic anhydrase inhibitor. Arch Ophthalmol 1993:111:1343.

7 Kitazawa Y, Azuma I, Iwata K, et al. Dorzolamide, a topical carbonic anhydrase inhibitor: A two-week dose response study in patients with glaucoma or ocular hypertension. J Glaucoma 1994;3:275.

8 Strahlman E, Tipping R, Vogel R. A Double masked, randomized 1-year study comparing dorzolamide (Trusopt), timolol, and betaxolol. The International Dorzolamide Study Group. Arch Ophthalmol 1995;113:1009-16.

9 Stewart WC, Sharpe ED, Harbin TS, et al. Brimonidine $0.2 \%$ versus dorzolamide $2 \%$ each given three times daily to reduce the intraocular pressure. Am J Ophthalmol 2000;129:723-7.

10 Book SA. Essentials of statistics. New York: McGraw Hill Book Company, 1978;117-22:205-15.

11 Mundorf TK, Cate EA, Sine CS, et al. The safety and efficacy of switching timolol maleate $0.5 \%$ solution to timolol hemihydrate $0.5 \%$ solution given twice daily. J Ocular Pharm and Therap 1998;14:129-35.

12 Duff GR. A double-masked crossover study comparing the effects of carteolol $1 \%$ and $2 \%$ on intraocular pressure. Acta Ophthalmologica 1987;65:618-21.

13 Stewart WC, Leland TM, Cate EA, et al. Efficacy and safety of timolol solution once daily vs. timolol gel in treating elevated IOP. J Glaucoma 1998;7:402-7.

14 Siegel S. Non-Parametric Statistics. Series in Psychology. New York: McGraw Hill Book Company, 1956:63-7.

15 Laibovitz R, Strahlman ER, Barber BL, et al. Comparison of quality of life and patient preference of dorzolamide and pilocarpine as adjunctive therapy to timolol in the treatment of glaucoma. J Glaucoma 1995;4:306-13.

16 Walters TR. Development and use of brimonidine in treating acute and chronic elevations of intraocular pressure: A review of safety, efficacy, dose response, and dosing studies. Surv Ophthalmol 1996;1:S19-S26.

17 LeBlanc RP. Twelve-month results of an ongoing randomized trial comparing brimonidine tartrate $0.2 \%$ and timolol $0.5 \%$ given twice daily in patients with glaucoma or ocular hypertension. Brimonidine Study Group 2. Ophthalmology 1998;105:1960-7.

18 Derick RJ, Walters TR, Robin AL, et al. Brimonidine tartrate: a one month dose response study. Invest Ophthalmol Vis Sci 1993;34:1138.

19 Nordland JR, Pasquale LR, Robin AL, et al. The cardiovascular, pulmonary, and ocular hypotensive effects of $0.2 \%$ brimonidine. Arch Ophthalmol 1995; 113:77-83.

20 Sherwood M, Brandt J, for the Bimatoprost Study Groups 1 and 2. Six-month comparison of bimatoprost once-daily with timolol twice-daily in patients with elevated intraocular pressures. Surv Ophthalmol 2001;45:S361-8.

21 Stewart WC, Holmes KT, Johnson MA. Washout periods for brimonidine $0.2 \%$ and latanoprost $0.005 \%$. Am J Ophthalmol 2001;131:798-9.

22 Stewart WC, Stewart JA, Day DG, et al. Efficacy and safety of timolol maleate /atanoprost fixed combination versus timolol maleate and brimonidine given twice daily. Acta Ophthalmol Scand 2003;81:242-6.

23 Boyle JE, Ghosh K, Gieser DK, et al. A randomized trial comparing the dorzolamide-timolol combination given twice daily to monotherapy with timolol and dorzolamide. Dorzolamide-Timolol Study Group. Ophthalmology 1998;105:1945-51

24 Clineshmidt CM, Williams RD, Snyder E, et al. A randomized trial in patients inadequately controlled on timolol alone comparing the dorzolamide-timolol combination to monotherapy with timolol or dorzolamide. Ophthalmology 1999; 106:17-24

25 Hutzelmann J, Owens S, Sedden A, et al. Comparison of the safety and efficacy of the fixed combination of dorzolamide/timolol and the concomitant administration of dorzolamide and timolol: A clinical equivalence study. International Clinical Equivalence Study Group. Br J Ophthalmol 1998:82:1249-53. 